\title{
Differentiation between recurrent gliomas and radiation necrosis using arterial spin labeling perfusion imaging
}

\author{
JING YE ${ }^{1}$, SANTOSH KUMAR BHAGAT ${ }^{1}$, HONGMEI LI $^{1}$, XIANFU LUO ${ }^{1}$, \\ BUHAI WANG ${ }^{2}$, LIQIN LIU ${ }^{2}$ and GUOMEI YANG ${ }^{1}$ \\ Departments of ${ }^{1}$ Radiology and ${ }^{2}$ Oncology, Northern Jiangsu People's Hospital, Yangzhou, Jiangsu 225001, P.R. China
}

Received November 11, 2014; Accepted November 25, 2015

DOI: $10.3892 /$ etm.2016.3225

\begin{abstract}
Arterial spin labeling (ASL) magnetic resonance (MR) perfusion imaging has been proposed as an effective method to measure brain tumor perfusion. The aim of the present study was to evaluate the utility of this technique in the differentiation of recurrent gliomas from radiation necrosis. Twenty-one patients with surgically treated primary gliomas, including 16 cases of recurrent glioma and 5 of radiation necrosis were examined using 3.0T MR imaging (MRI). ASL and dynamic susceptibility contrast-weighted (DSC) perfusion MRI scans were performed. Maps of normalized cerebral blood flow (CBF) in ASL imaging and cerebral blood volume (CBV) in DSC imaging were computed and analyzed in the regions of interest. In cases of glioma recurrence, the normalized ASL-CBF ratio (4.45 \pm 2.72$)$ was higher than that in cases of radiation injury $(1.22 \pm 0.61)(\mathrm{P}<0.01)$. The normalized DSC-relative CBV ratio was also significantly higher in glioma recurrence $(3.38 \pm 2.08)$ than it was in radiation injury $(1.09 \pm 0.55)(\mathrm{P}<0.05)$. A close linear correlation was found between the ASL and DSC MRI techniques (linear regression coefficient, $\mathrm{R}=0.85 ; \mathrm{P}=0.005)$ in the differentiation of recurrent glioma from radiation injury. The results indicate that ASL perfusion is an accurate method of distinguishing between glioma recurrence and radiation necrosis.
\end{abstract}

\section{Introduction}

Radiation therapy is one of the main therapeutic methods used in the treatment of gliomas following surgical excision, despite the fact that it often causes radiation necrosis (reported incidence rate, $2-24 \%$ ), the most severe type of radiation injury $(1,2)$. Following treatment, frequent monitoring is

Correspondence to: Professor Liqin Liu, Department of Oncology, Northern Jiangsu People's Hospital, 98 Nantong West Road, Yangzhou, Jiangsu 225001, P.R. China

E-mail: yzhyejing@hotmail.com

Key words: magnetic resonance imaging, glioma, recurrence, radiation injuries required for the evaluation of the therapy, and magnetic resonance imaging (MRI) is the preferred modality.

Glioma growth is often accompanied by the breakdown of the blood brain barrier (BBB) and a higher cerebral blood volume (CBV), which is due to tumor angiogenesis $(3,4)$; however, BBB leakage also occurs in radiation injury. Both types of lesions appear hyperintense on T2-weighted images and show strong contrast enhancement with surrounding edema and mass effect, which makes it impossible to differentiate between glioma recurrence and radiation injury using the conventional enhanced MRI (5-7); therefore, there is an urgent requirement for the development of new, functional imaging modalities for the evaluation of the effectiveness of glioma treatment.

Dynamic susceptibility contrast-weighted (DSC) magnetic resonance (MR) perfusion imaging allows for the measurement of cerebral blood flow (CBF) and CBV, which are known to correlate with both the histologic tumor grade and individual histological features (8-10). A previous study has shown that DSC-MRI is useful for the diagnosis of glioma recurrence and radiation necrosis (11). However, there are several disadvantages to using this technique. First, it requires an intravenous injection of a gadolinium contrast agent $(12,13)$. Secondly, in DSC-MRI, contrast agent extravasation causes T2-weighted signal intensity loss, which can in turn result in a decreased relative CBV (rCBV) for high-grade tumors (14). Thirdly, this technique is mainly based on gradient-echo or echo planar imaging (EPI), which have been shown to be highly sensitive to susceptibility, which may decrease the rCBV ratio (15).

Arterial spin labeling (ASL) MR perfusion imaging utilizes labeled blood water as the endogenous tracer, and is recognized as a non-invasive method of measuring CBF. Several studies have used this technique to evaluate glioma grading and tumor angiogenesis (16-18). The aim of the present study was to assess the ability of ASL perfusion imaging to differentiate between glioma recurrence and radiation necrosis and compare it with the DSC-MRI technique.

\section{Materials and methods}

Subjects. The present study was approved by the Ethics Committee of the Northern Jiangsu Province Hospital (Yangzhou, China). 
Between March 2012 and March 2014, 57 surgically treated patients with pathologically confirmed primary gliomas underwent follow-up MRI. Using contrast-enhanced T1-weighted imaging, contrast-enhancing lesions were observed in 21 of these cases and were included in the present study. Sixteen patients received radiotherapy with 50-60 Gy, while 5 received radiochemotherapy (50-60 Gy) combined with temozolomide. Patients to whom temozolomide was administered concurrently received a daily temozolomide dosage of $75 \mathrm{mg} / \mathrm{m}^{2}$ during intensity-modulated radiation therapy, and then at $150 \mathrm{mg} / \mathrm{m}^{2}$ for 5 days in each of six 28 -day treatment cycles.

Follow-up MRI scans were performed every 3 months and included T2-weighted, diffusion weighted (DW) and ASL perfusion imaging, followed by DSC-MRI.

Materials and methods. Conventional MR images were acquired using a 3.0T MR imaging system (Discovery MR750; GE Healthcare, Fairfield, CT, USA) with a 16-channel coil specifically for imaging the head. Conventional sequences included axial, sagittal and coronal T2-weighted, axial T1-weighted, fluid-attenuated inversion recovery sequence and DW imaging.

ASL imaging used a 3-dimensional (3D) pseudocontinuous ASL method and was performed prior to DSC-MRI as follows: Repetition time (TR), 4,632 msec; echo time (TE), $1.5 \mathrm{msec}$; acquisition matrix, 96x61; field of view (FOV), $24 \mathrm{~cm}$; slice thickness, $4 \mathrm{~mm}$; interslice gap, $0 \mathrm{~mm}$ and post label delay, $1,535 \mathrm{msec}$ with spiral acquisition along with 3D proton density-weighted fast spin echo (FSE) EPI sequence as follows: TR, $3.9 \mathrm{msec}$, TE, $1.9 \mathrm{msec}$, parallel imaging factor, 2; acquisition matrix, 96x61; FOV, $24 \mathrm{~cm}$; slice thickness, $4 \mathrm{~mm}$ and interslice gap, $0 \mathrm{~mm}$. The dosage of contrast agent (Magnevist; Bayer HealthCare Pharmaceuticals, Berlin, Germany) was $0.05 \mathrm{mmol} / \mathrm{kg}$. The automatic bolus injection (Medrad Spectris Solaris; Bayer HealthCare, Saxonburg, PA, USA) of the contrast agent (rate, $3 \mathrm{ml} / \mathrm{sec}$ ) was followed by an injection of $20 \mathrm{ml}$ saline. The total acquisition time for perfusion measurement and bolus tracking was 1:20 min.

\section{Image evaluation}

Qualitative assessment. All images were reviewed by two neuroradiologists who were blinded to the clinical and histopathological history of the cases. The images were evaluated using the FuncTool Performance software package with the Advantage 4.5 Workstation (GE Healthcare).

Since the regions of the gliomas with maximum perfusion are suggestive of malignancy and aggressiveness, three maximally perfusion regions of the entire lesions were located by drawing regions of interest (ROIs) of area $0.5-2.0 \mathrm{~cm}^{2}$. The average of the values from these regions was calculated and normalized to the contralateral normal white matter.

Artifacts. A 5-point scale was used to assess the image artifacts (ranging from 1, severe degradation to 5, little degradation) caused by motion and susceptibility effects. A score of 5 was attributed to an excellent image quality, with almost no artifacts; while a score of 3 indicated degraded image quality owing to artifacts (sufficiently degraded to interfere with accurate diagnosis).
Table I. Artifact scores in ASL and DSC imaging.

$$
\text { Artifact score (mean } \pm \text { SD) }
$$

\begin{tabular}{lcc}
\cline { 2 - 3 } Imaging & Motion artifact & Susceptibility artifact \\
\hline ASL-CBF & $4.75 \pm 0.44$ & $4.85 \pm 0.37$ \\
DSC-CBV & $4.60 \pm 0.50$ & $4.15 \pm 0.75$ \\
P-value & 0.508 & 0.0004 \\
\hline
\end{tabular}

ASL, arterial spin labeling; CBF, cerebral blood flow; DSC, dynamic susceptibility contrast-weighted; CBV, cerebral blood flow; $\mathrm{SD}$, standard deviation.

Classification of lesions. Six cases of glioma recurrence were classified according to the histopathological analysis, while 15 lesions (10 lesions of glioma recurrence and 5 of radiation necrosis) were verified based on the follow-up MRI scan according to the Macdonald criteria $(19,20)$. Each patient was followed up for a minimum of 11 months, and the rate of follow-up was $100 \%$.

Statistical analysis. All statistical analyses were performed using SPSS 16.0 (SPSS, Inc., Chicago, IL, USA). The unpaired Student's t-test was used for the comparison between the $\mathrm{CBF}$ and $\mathrm{rCBV}$ values of recurrent gliomas and those radiation necrosis. Artifact scores in ASL and DSC images were compared using Wilcoxon's sign-rank test. Linear regression analysis was used to evaluate the correlation between ASL-CBF and DCS-CBV. $\mathrm{P}<0.05$ was considered to indicate a statistically significant difference.

\section{Results}

Patient diagnosis and imaging results. The present study included 21 patients (11 men and 10 women), with an age range of 32-63 years and a mean age of 51.3 years. Six cases of glioma recurrence were confirmed by surgical pathology or biopsy, and 10 cases of glioma recurrence were verified based on the Macdonald criteria (19), since they showed clear radiological evidence of disease progression despite the use of therapy. Radiation necrosis was diagnosed in 5 cases by follow-up MRI, in which enhanced lesions disappeared or decreased in size without any treatment.

Representative images of patients with glioma recurrence and radiation injury are presented in Figs. 1 and 2. Analysis of the images revealed that glioma recurrence exhibited a higher normalized ASL-CBF ratio $(4.45 \pm 2.72)$ compared with that of radiation injury $(1.22 \pm 0.61)(\mathrm{P}<0.01)$. In addition, the normalized DSC-rCBV ratio in glioma recurrence $(3.38 \pm 2.08)$ was significantly higher than that in radiation injury $(1.09 \pm 0.55)$ $(\mathrm{P}<0.05)$.

Linear regression analysis. As demonstrated in Fig. 3, the linear regression analysis revealed that there was a close correlation between normalized ASL-CBF and normalized DSC-CBV, with $\mathrm{R}=0.85$ and an equation of $\mathrm{y}=0.56+1.15 \mathrm{x}$ for the regression line, which was statistically different from identity at $\mathrm{P}<0.05$. 


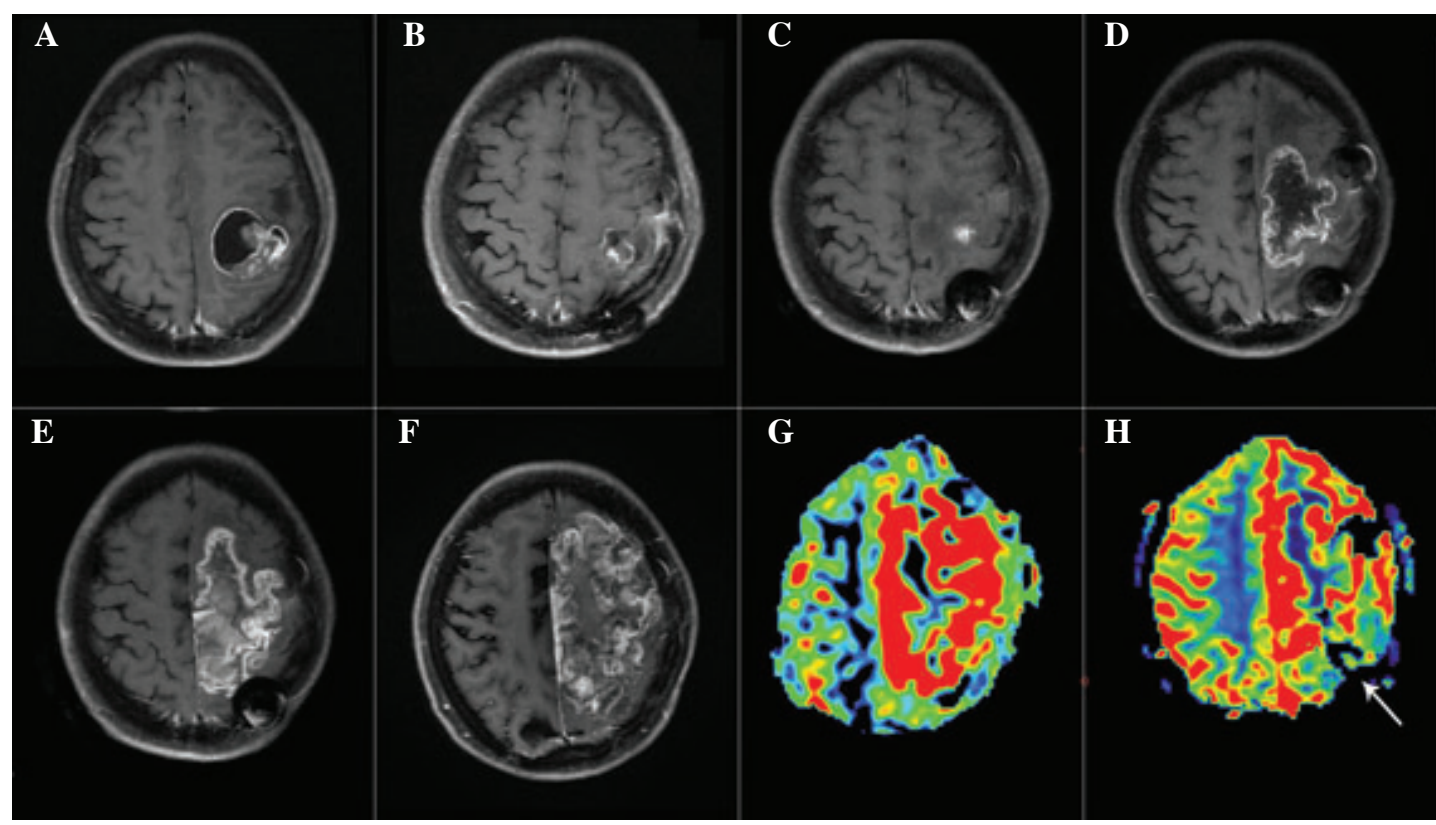

Figure 1. Glioma recurrence in a 54-year-old male patient with glioblastomas. T1-weighted images of the glioblastomas (A) pre-surgically, (B) post-surgically and at the (C) 3-, (D) 6-, (E) 10- and (F) 14-month follow-ups. (G) Arterial spin labeling-cerebral blood flow and (H) dynamic susceptibility contrast-weighted-cerebral blood volume imaging at the 14-month follow-up. The follow-up magnetic resonance imaging scan performed 6 months after the administration of radiation therapy with temozolomide shows an increase in lesion size. (H) Note the evident magnetic susceptibility artifact near the operation area leading to an underestimation of tumor perfusion (white arrow).



Figure 2. Radiation injury in a 37-year-old man with glioblastoma multiforme. T1-weighted, post-surgical images at (A) 9 , (B) 12 , (C) 16 and (D) 24 months . (E) Arterial spin labeling-cerebral blood flow (ASL-CBV) and (F) dynamic susceptibility contrast-weighted-cerebral blood volume (DSC-CBV) images at 12 months. The follow-up magnetic resonance imaging scans show a reduction in lesion size. (E and F) Low perfusion in the enhanced area of the T1-weighted images is shown by ASL-CBF (white arrow) and DSC-CBV imaging.

Artifact scores. Artifacts in the ASL-CBF and DSC-CBV images were scored as follows (Table I): Motion artifact, $4.75 \pm 0.44$ and $4.60 \pm 0.50$, respectively $(\mathrm{P}=0.508)$; susceptibility artifact, $4.85 \pm 0.37$ and $4.15 \pm 0.75$, respectively $(\mathrm{P}<0.01)$. No case scored $\leq 3$ due to motion artifacts in either of the two imaging techniques or due to susceptibility artifacts in the measurement of ASL-CBF. With regard to the estimation of DSC-rCBV, 5 cases scored 3, due to susceptibility artifacts.

\section{Discussion}

In the present study, the ability of the ASL technique to differentiate between glioma recurrence and radiation necrosis 


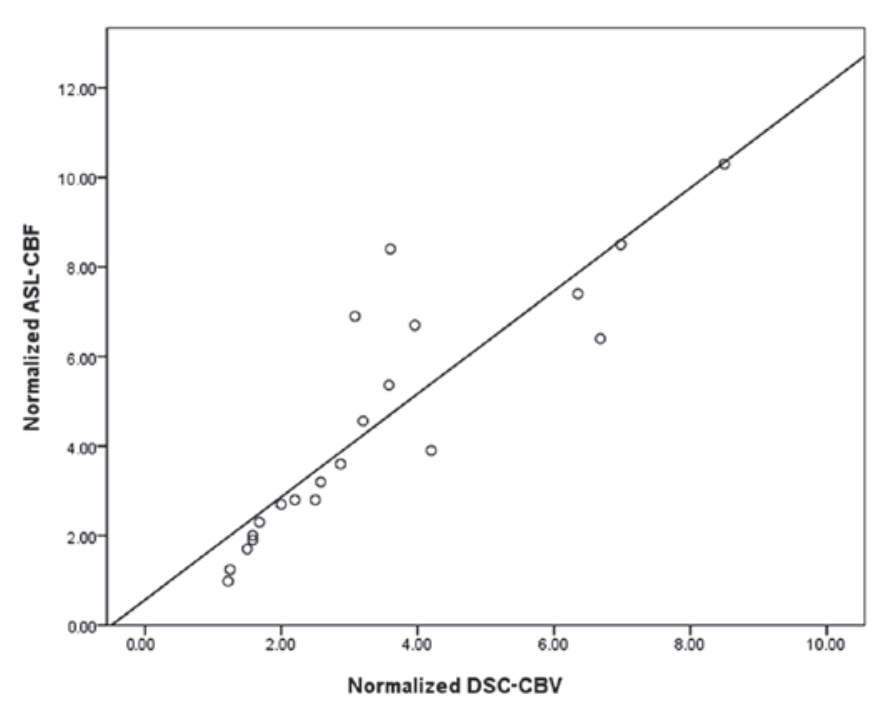

Figure 3. Scatterplot showing normalized ASL-CBF and DCS-CBV in all cases. Linear regression analysis revealed a high correlation between the two values, with $\mathrm{R}=0.85$ and a formula of $\mathrm{y}=0.56+1.15 \mathrm{x}$ for the regression line, which is statistically different from identity at $\mathrm{P}<0.05$. ASL-CBF, arterial spin labeling-cerebral blood flow; DCS-CBV, dynamic susceptibility contrast-weighted-cerebral blood volume.

was evaluated. The results demonstrated that there is a close correlation between the ASL and DSC methods with regard to distinguishing between the two conditions.

Perfusion imaging of brain tumors, which mainly includes DSC-MR perfusion techniques, has been used for tumor grading, guiding tumor biopsy and assessing the response to treatment (21). However, the use of an exogenous contrast agent is a major limitation in the routine clinical application of this method, since contrast agent extravasation and high sensitivity to susceptibility can result in a decreased rCBV for high-grade tumors (9). ASL imaging constitutes another MR perfusion method, which is used for the assessment of brain tumor vascularity (22). In this technique, the contrast agent used is labeled arterial blood water proximal to the brain. Since $90 \%$ of the labeled water passes through the capillary bed on the first pass and T1 decay is considerably shorter than the capillary transit time, contrast agent extravasation and dispersion do not interfere with ASL signal intensity (19,23). In addition, since no exogenous contrast agent is required, ASL imaging could be ideal for the long-term follow-up of gliomas following radiation, including those with renal dysfunction.

Previous studies have shown that ASL imaging could potentially differentiate between glioma recurrence and radiation injury. The study conducted by Ozsunar et al (24) demonstrated that ASL imaging could accurately distinguish predominant recurrent high-grade glioma from radiation necrosis; however, the results were based on a single-slice method with a scanning time of 4-8 min, dependent on lesion size, which limited the wide range of clinical applications. Choi et al (25) showed the diagnostic superiority of combined ASL and DSC perfusion compared with DSC imaging alone in the differentiation of pseudoprogression from early tumor progression, with ASL having a lower sensitivity than DSC perfusion. There are, however, differences between the study of Choi et al (25) and the present study. ASL imaging in the previous study was based on an gradient-echo sequence, which is more vulnerable to magnetic susceptibility artifacts. Furthermore, Choi et al only assessed the diagnostic performance of DCS perfusion alone or combined with ASL imaging, while the present study aimed to assess the diagnostic performance of ASL imaging. The results of the present study suggest that there is a close correlation between ASL perfusion imaging and DSC-MRI (Fig. 3) and that ASL-CBF could effectively distinguish glioma recurrence from radiation injury.

DSC-MRI was shown to exhibit a high sensitivity to susceptibility, due to magnetic susceptibility artifacts, which could lead to an underestimation of tumor perfusion when the ROI is close to surgically treated regions of the brain or areas affected by bleeding $(26,27)$. In the present study, however, the ASL imaging was conducted utilizing the 3D FSE technique, which features high spatial resolution and reduced magnetic susceptibility. No evident magnetic susceptibility artifacts were found to influence the image quality in the present study, indicating that this ASL imaging method is suitable for the follow-up of glioma after surgical excision.

The study did, however, have several limitations. First, the sample size was rather small, particularly that of patients with radiation injury, which does not allow for generalization of the present findings; further research using a larger population is required. Secondly, only the most clearly enhanced regions were analyzed; however, edema in a portion of the glioma may represent tumor infiltration, the identification of which may contribute to an improved evaluation of the tumor. Finally, in the ASL imaging, only a single delay time between labeling and imaging was used, which may result in an inaccurate estimation of the CBF, due to differences in the cerebral circulation among individuals.

In conclusion, the aforementioned findings demonstrate the potential of $\mathrm{CBF}$ as determined by ASL perfusion imaging in the differentiation of glioma recurrence from radiation injury. ASL imaging could potentially be used to determine the perfusion patterns in patients with surgically treated primary gliomas, and could also prove useful in the selection of the appropriate treatment option.

\section{Acknowledgements}

This study was supported by The National Natural Science Fund of China (grant no. 81371377).

\section{References}

1. Tsuruda JS, Kortman KE, Bradley WG, Wheeler DC, Van Dalsem W and Bradley TP: Radiation effects on cerebral white matter: MR evaluation. AJR Am J Roentgenol 149: 165-171, 1987.

2. Remler MP, Marcussen WH and Tiller-Borsich J: The late effects of radiation on the blood brain barrier. Int J Radiat Oncol Biol Phys 12: 1965-1969, 1986.

3. Leon SP, Folkerth RD and Black PM: Microvessel density is a prognostic indicator for patients with astroglial brain tumors. Cancer 77: 362-372, 1996.

4. Huang AP, Tsai JC, Kuo LT, Lee CW, Lai HS, Tsai LK, Huang SJ, Chen CM, Chen YS, Chuang HY and Wintermark M: Clinical application of perfusion computed tomography in neurosurgery. J Neurosurg 120: 473-488, 2014.

5. Mullins ME, Barest GD, Schaefer PW, Hochberg FH, Gonzalez RG and Lev MH: Radiation necrosis versus glioma recurrence: Conventional MR imaging clues to diagnosis. AJNR Am J Neuroradiol 26: 1967-1972, 2005. 
6. Brandes AA, Tosoni A, Spagnolli F, Frezza G, Leonardi M, Calbucci $F$ and Franceschi E: Disease progression or pseudoprogression after concomitant radiochemotherapy treatment: Pitfalls in neurooncology. Neuro Oncol 10: 361-367, 2008.

7. Kumar AJ, Leeds NE, Fuller GN, Van Tassel P, Maor MH, Sawaya RE and Levin VA: Malignant gliomas: MR imaging spectrum of radiation therapy- and chemotherapy-induced necrosis of the brain after treatment. Radiology 217: 377-384, 2000

8. Cha S, Knopp EA, Johnson G, Wetzel SG, Litt AW and Zagzag D: Intracranial mass lesions: Dynamic contrast-enhanced susceptibility-weighted echo-planar perfusion MR imaging. Radiology 223: 11-29, 2002.

9. Paulson ES and Schmainda KM: Comparison of dynamic susceptibility-weighted contrast-enhanced MR methods Recommendations for measuring relative cerebral blood volume in brain tumors. Radiology 249: 601-613, 2008.

10. Thomsen H, Steffensen E and Larsson EM: Perfusion MRI (dynamic susceptibility contrast imaging) with different measurement approaches for the evaluation of blood flow and blood volume in human gliomas. Acta Radiol 53: 95-101, 2012.

11. Barajas RF Jr, Chang JS, Segal MR, Parsa AT, McDermott MW, Berger MS and Cha S. Differentiation of recurrent glioblastoma multiforme from radiation necrosis after externa beam radiation therapy with dynamic susceptibility-weighted contrast-enhanced perfusion MR imaging. Radiology 253: 486-496, 2009.

12. Matsumura T, Hayakawa M, Shimada F, Yabuki M, Dohanish S, Palkowitsch P and Yoshikawa K: Safety of gadopentetate dimeglumine after 120 million administrations over 25 years of clinical use. Magn Reson Med Sci 12: 297-304, 2013.

13. Yang L, Krefting I, Gorovets A, Marzella L, Kaiser J, Boucher R and Rieves D: Nephrogenic systemic fibrosis and class labeling of gadolinium-based contrast agents by the food and drug administration. Radiology 265: 248-253, 2012.

14. Bjornerud A and Emblem KE: A fully automated method for quantitative cerebral hemodynamic analysis using DSC-MRI. J Cereb Blood Flow Metab 30: 1066-1078, 2010.

15. Carlsson A, Starck G, Ljungberg M, Ekholm S and Forssell-Aronsson E: Accurate and sensitive measurements of magnetic susceptibility using echo planar imaging. Magn Reson Imaging 24: 1179-1185, 2006.

16. Detre JA, Rao H, Wang DJ, Chen YF and Wang Z: Applications of arterial spin labeled MRI in the brain. J Magn Reson Imaging 35: 1026-1037, 2012.

17. Detre JA, Wang J, Wang Z and Rao H: Arterial spin-labeled perfusion MRI in basic and clinical neuroscience. Curr Opin Neuro 22: 348-355, 2009 .
18. Chawla S, Wang S, Wolf RL, Woo JH, Wang J, O'Rourke DM, Judy KD, Grady MS, Melhem ER and Poptani H: Arterial spin-labeling and MR spectroscopy in the differentiation of gliomas. AJNR Am J Neuroradiol 28: 1683-1689, 2007.

19. Macdonald DR, Cascino TL, Schold SC Jr and Cairncross JG: Response criteria for phase II studies of supratentorial malignant glioma. J Clin Oncol 8: 1277-1280, 1990.

20. Tan H, Chen L, Guan Y and Lin X: Comparison of MRI, F-18 FDG and 11C-choline PET/CT for their potentials in differentiating brain tumor recurrence from brain tumor necrosis following radiotherapy. Clin Nucl Med 36: 978-981, 2011.

21. Sugahara T, Korogi Y, Tomiguchi S, Shigematsu Y, Ikushima I, Kira T, Liang L, Ushio Y and Takahashi M: Posttherapeutic intraaxial brain tumor: The value of perfusion-sensitive contrast-enhanced MR imaging for differentiating tumor recurrence from nonneoplastic contrast-enhancing tissue. AJNR Am J Neuroradiol 21: 901-909, 2000.

22. Warmuth C, Gunther M and Zimmer C: Quantification of blood flow in brain tumors: Comparison of arterial spin labeling and dynamic susceptibility-weighted contrast-enhanced MR imaging. Radiology 228: 523-532, 2003.

23. White CM, Pope WB, Zaw T, Qiao J, Naeini KM, Lai A, Nghiemphu PL, Wang JJ, Cloughesy TF and Ellingson BM: Regional and voxel-wise comparisons of blood flow measurements between dynamic susceptibility contrast magnetic resonance imaging (DSC-MRI) and arterial spin labeling (ASL) in brain tumors. J Neuroimaging 24: 23-30, 2014.

24. Ozsunar Y, Mullins ME, Kwong K, Hochberg FH, Ament C, Schaefer PW, Gonzalez RG and Lev MH: Glioma recurrence versus radiation necrosis? A pilot comparison of arterial spin-labeled, dynamic susceptibility contrast enhanced MRI and FDG-PET imaging. Acad Radiol 17: 282-290, 2010.

25. Choi YJ, Kim HS, Jahng GH, Kim SJ and Suh DC: Pseudoprogression in patients with glioblastoma: Added value of arterial spin labeling to dynamic susceptibility contrast perfusion MR imaging. Acta Radiol 54: 448-454, 2013.

26. Hu LS, Baxter LC, Smith KA, Feuerstein BG, Karis JP, Eschbacher JM, Coons SW, Nakaji P, Yeh RF, Debbins J and Heiserman JE: Relative cerebral blood volume values to differentiate high-grade glioma recurrence from posttreatment radiation effect: Direct correlation between image-guided tissue histopathology and localized dynamic susceptibility-weighted contrast-enhanced perfusion MR imaging measurements. AJNR Am J Neuroradiol 30: 552-558, 2009.

27. Larsen VA, Simonsen HJ, Law I, Larsson HB and Hansen AE Evaluation of dynamic contrast-enhanced T1-weighted perfusion MRI in the differentiation of tumor recurrence from radiation necrosis. Neuroradiology 55: 361-369, 2013. 